\title{
Metabolic Health Has Greater Impact on Diabetes than Simple Overweight/Obesity in Mexican Americans
}

\author{
Shenghui Wu, ${ }^{1}$ Susan P. Fisher-Hoch, ${ }^{2}$ Belinda Reninger, ${ }^{2}$ \\ Kristina Vatcheva, ${ }^{2}$ and Joseph B. McCormick ${ }^{2}$ \\ ${ }^{1}$ Department of Epidemiology \& Biostatistics, University of Texas Health Science Center at San Antonio, Laredo Campus, \\ Laredo, TX 78045, USA \\ ${ }^{2}$ Division of Epidemiology, School of Public Health, University of Texas Health Science Center at Houston, \\ Brownsville Campus, Brownsville, TX 78520, USA
}

Correspondence should be addressed to Joseph B. McCormick; joseph.b.mccormick@uth.tmc.edu

Received 12 August 2015; Revised 13 October 2015; Accepted 15 October 2015

Academic Editor: Ed Randell

Copyright (C) 2016 Shenghui Wu et al. This is an open access article distributed under the Creative Commons Attribution License, which permits unrestricted use, distribution, and reproduction in any medium, provided the original work is properly cited.

\begin{abstract}
Purpose. To compare the risk for diabetes in each of 4 categories of metabolic health and BMI. Methods. Participants were drawn from the Cameron County Hispanic Cohort, a randomly selected Mexican American cohort in Texas on the US-Mexico border. Subjects were divided into 4 phenotypes according to metabolic health and BMI: metabolically healthy normal weight, metabolically healthy overweight/obese, metabolically unhealthy normal weight, and metabolically unhealthy overweight/obese. Metabolic health was defined as having less than 2 metabolic abnormalities. Overweight/obese status was assessed by BMI higher than $25 \mathrm{~kg} / \mathrm{m}^{2}$. Diabetes was defined by the 2010 ADA definition or by being on a diabetic medication. Results. The odds ratio for diabetes risk was 2.25 in the metabolically healthy overweight/obese phenotype (95\% CI 1.34, 3.79), 3.78 (1.57, 9.09) in the metabolically unhealthy normal weight phenotype, and $5.39(3.16,9.20)$ in metabolically unhealthy overweight/obese phenotype after adjusting for confounding factors compared with the metabolically healthy normal weight phenotype. Conclusions. Metabolic health had a greater effect on the increased risk for diabetes than overweight/obesity. Greater focus on metabolic health might be a more effective target for prevention and control of diabetes than emphasis on weight loss alone.
\end{abstract}

\section{Introduction}

The proportion of overweight/obese adults in the US increased between 1980 and 2013 from $28.8 \%$ to $36.9 \%$ in men and from $29.8 \%$ to $38.0 \%$ in women [1]. Overweight/obesity increases the risk for type 2 diabetes mellitus [2,3]. However, being metabolically unhealthy also increases the risk for type 2 diabetes [4-6]. Though these findings suggest that the risk for type 2 diabetes associated with overweight/obesity is influenced by the coexistence of metabolic abnormalities, the independent impact of these two conditions is unclear. Metabolically unhealthy normal weight (MUHNW) subjects are individuals with normal weight but with metabolic abnormalities [7, 8], but the phenotype is ill-defined. Among normal weight individuals aged 20 years or older in the Third National Health and Nutrition Examination Survey, 4.6\% of men and $6.2 \%$ of women had three or more metabolic abnormalities [9]. Because MUHNW subjects are not overweight or obese, they may not be aware of their risks and may be missed and therefore may not benefit from adequate prevention. Nevertheless, MUHNW carries a significant risk for cardiovascular diseases $[10,11]$ and mortality [12]. The findings from several studies have shown the increased risk of diabetes in MUHNW individuals [4-6] compared with metabolically healthy normal weight (MHNW) individuals in different ethnicities, but only one study was conducted in Mexican Americans [6].

There is no consensus on the risk presented by the metabolically healthy overweight/obese (MHOW) phenotype $[4,5]$. Evidence regarding the risk of diabetes associated with the MHOW phenotype is also uncertain. It has been reported that MHOW individuals may be more likely to develop incident diabetes compared with normal weight individuals [4-6]; however data from Kangbuk Samsung Health 
Study of more than 6 thousand individuals did not show a significant association with diabetes [5] and data in Mexican Americans is in any event limited [6]. Although metabolically unhealthy overweight/obese (MUHOW) subjects showed a higher risk of diabetes than MHNW phenotype [4-6, 13], only a few studies compared the diabetes risk between MUHOW, MUHNW, MHOW, and MHNW phenotypes [46] and only one was conducted in Mexican Americans [6].

Mexican Americans have higher prevalence of diabetes, overweight/obesity, and metabolic disturbances than nonHispanic Whites [14-16]. The objective of this study was to compare the risk for diabetes among the 4 phenotypes divided by metabolic health and overweight/obesity status in a randomly selected cohort of Mexican American subjects.

\section{Materials and Methods}

2.1. Study Participants. This study was approved by the Committee for the Protection of Human Subjects of the UT Health, Houston and the Institutional Review Board of the University of the Texas Health Science Center, San Antonio. All study participants gave written informed consent. This cross-sectional analysis used data from the Cameron County Hispanic Cohort (CCHC), a homogenous community-dwelling Mexican American ongoing cohort study $[17,18]$. Study subjects were recruited from randomly selected blocks according to the 2000 Census as described previously $[17,18]$. At the baseline survey conducted between 2003 and 2014, 3,257 participants aged 18 years or older were recruited from their households in predominantly Mexican American cities along the Rio Grande border with Mexico. To reduce the effect of type I diabetes on the results, the participants who had diabetes before 18 years were excluded $(n=10)$.

All subjects responded to a detailed baseline survey of demographic characteristics, lifestyle including diet, physical activity, family, and medical history, and other exposures. Participants were asked to fast for at least 10 hours overnight before a clinic visit at the clinical research unit. Anthropometric measurements, including current weight, height, and circumferences of the waist and hip, were also taken $[17,18]$.

Weight was measured to the nearest tenth of a kilogram and height to the nearest tenth of a centimeter. Body mass index (BMI) was calculated as weight in kilograms divided by height squared in meters $\left(\mathrm{kg} / \mathrm{m}^{2}\right)$. Waist circumference (WC) was measured at the level of the umbilicus and hip circumference (HC) at the level of maximum width of the buttocks with participants in a standing position and breathing normally, to the nearest $0.2 \mathrm{~cm}$. Waist-to-hip ratio (WHR) was calculated as WC divided by HC [17]. Body fat percentage was estimated using the resistance values from the Quantum X bioelectric body composition analyzer with the sex-specific equations from Sun et al. [19]. The average of 3 blood pressure (BP) measurements taken 5 minutes apart were used.

All participants completed a detailed baseline survey that collected information on demographic characteristics, lifestyle and dietary histories, medical history, and other exposures. Physical activity was assessed using the International Physical Activity Questionnaire (IPAQ) short form [20]; reported minutes of physical activity per week were weighted by a metabolic equivalent (MET, multiples of resting energy expenditure) resulting in a physical activity estimate expressed as MET-minutes per week [20]. Physical activity energy expenditure was estimated using standard metabolic equivalent (MET) values [20].

2.2. Laboratory Measurements. All participants donated a blood sample at baseline. After collection, samples were placed on ice and centrifuged within 30 minutes of collection. Following processing and aliquoting, all samples were stored at $-80^{\circ} \mathrm{C}$ until laboratory analyses were conducted. Laboratory studies performed included fasting lipid panel, hemoglobin $(\mathrm{Hb}) \mathrm{Alc}$, fasting plasma glucose, and fasting serum insulin. Homeostasis model assessment insulin resistance index (HOMA-IR) was calculated as fasting glucose $(\mathrm{mg} / \mathrm{dL}) / 18 \times$ fasting insulin $(\mathrm{mU} / \mathrm{L}) / 22.5$ [21]. High sensitivity C-reactive protein (CRP) levels were measured using Quantikine ELISA kit (R \& D Systems, Inc., Minneapolis, USA).

2.3. Identification of the Overweight/Obese and Metabolic Health. Participants were categorized as overweight/obese or with normal weight using a BMI cutoff of $25.0 \mathrm{~kg} / \mathrm{m}^{2}$ [1] and then were further categorized as metabolically healthy or unhealthy. Metabolic health was defined as having $<2$ of the following metabolic abnormalities: systolic BP (SBP) $\geq 130 \mathrm{mmHg}$ and/or diastolic $\mathrm{BP}(\mathrm{DBP}) \geq 85 \mathrm{mmHg}$ or on antihypertensive medication; triglyceride $\geq 150 \mathrm{mg} / \mathrm{dL}$; high-density lipoprotein cholesterol $<40 \mathrm{mg} / \mathrm{dL}$ in men or $<50 \mathrm{mg} / \mathrm{dL}$ in women; or HOMA-IR value $>90$ th percentile $[22,23]$. Waist circumference was not included due to its high correlation with BMI [22]. To avoid bias we did not use blood glucose levels nor diabetes medication in the definition of metabolic health so as to compare the risk for diabetes in 4 phenotypes of metabolic health and BMI.

According to the above criteria, participants were divided into four phenotypes:

(1) MHNW: metabolically healthy, normal weight: BMI $<25 \mathrm{~kg} / \mathrm{m}^{2}$ and $<2$ metabolic risk factor;

(2) MHOW: metabolically healthy, overweight/obese: $\mathrm{BMI} \geq 25 \mathrm{~kg} / \mathrm{m}^{2}$ and $<2$ metabolic risk factor;

(3) MUHNW: metabolically unhealthy, normal weight: $\mathrm{BMI}<25 \mathrm{~kg} / \mathrm{m}^{2}$ and $\geq 2$ metabolic risk factor;

(4) MUHOW: metabolically unhealthy, overweight/ obese: $\mathrm{BMI} \geq 25 \mathrm{~kg} / \mathrm{m}^{2}$ and $\geq 2$ metabolic risk factor.

2.4. Identification of Diabetes. Diabetes was identified by the 2010 definition of diabetes of the American Diabetes Association [24] or the participants reporting being told by a health care provider that they had diabetes or if they were taking hypoglycemic medication. 
2.5. Statistical Analysis. Descriptive results and the models reported in this paper were adjusted for the probability of sampling using weights taking into consideration clustering effects arising from the census block and household [17]. Logtransformation was conducted to normalize the distribution of the biomarkers studied as appropriate. Survey-weighted linear regression was used to obtain the $t$-test statistics to compare phenotypes and to be used for multiple pairwise mean comparisons for continuous data. Survey-weighted chisquare test was used to obtain Rao-Scott $F$ adjusted chisquare statistic to compare phenotypes for categorical data. Survey-weighted logistic regression modeling was performed to estimate the odds ratios (ORs) for diabetes risk and their $95 \%$ confidence intervals (CIs) by the metabolic health and overweight/obese phenotype phenotypes adjusting for other covariates. Initially, a multivariable survey-weighted logistic regression model was created to identify independent factors associated with diabetes, among variables including the overweight/obese phenotypes, age, gender, education, reported minutes of physical activity per week, servings of fruits and vegetables per day, and alcohol drinking and cigarette smoking status. Variables that were not significant and were not confounders were excluded from the final model. The interaction effects between the independent variables were tested. The analysis involved in physical activity and dietary data was conducted in 2,044 participants because the interview was only administered in these subjects.

To compare the risk of diabetes in different metabolic health and overweight/obese phenotypes, we also used a restricted cubic spline logistic regression analysis [25] to evaluate the risk of diabetes with age $(P<0.0001$ for all participants) stratified by metabolic health and overweight/obese phenotypes. Knots were placed at the 5th, 50th, and 95th percentiles of the distribution of age at enrollment. We excluded participants whose age at enrollment was below 20 or above 70 from the restricted cubic spline model to minimize the influence of outliers.

Sensitivity analyses were performed including fasting glucose $>100 \mathrm{mg} / \mathrm{dL}$ or on hypoglycemic medication as a component for the definition of metabolic health (metabolically healthy, 0 metabolic abnormalities; metabolically unhealthy, $\geq 2$ metabolic abnormalities) [22, 23]. Participants were categorized as overweight $\left(25-29.9 \mathrm{~kg} / \mathrm{m}^{2}\right)$ or obese $\left(\geq 30 \mathrm{~kg} / \mathrm{m}^{2}\right)$ using BMI cutoffs of 25.0 and $30 \mathrm{~kg} / \mathrm{m}^{2}$ [1] and separate analyses by overweight and obesity were also conducted. Statistical analyses were carried out by using SAS version 9.3 (SAS Institute, Cary, NC). All statistical tests were based on two-sided probability.

\section{Results}

At the time of this study a total of 3,257 individuals were enrolled in the CCHC, 2,893 participants from Brownsville and 242 participants from Harlingen (Lower Rio Grande Valley) and 138 participants from Laredo (Webb County), Texas. Among 3,247 remaining participants after excluding 10 subjects who developed diabetes before 18 years of age (to minimize potential type I diabetes), 475 subjects (14.6\%) were classified as MHNW, 1,594 (49.1\%) as MHOW, $72(2.2 \%)$ as
MUHNW, and 1,106 (34.1\%) as MUHOW (Table 1). Mean age of this subset was 46 years; $34 \%$ were male. A total of $36.3 \%(n=1,178)$ were classified as metabolically unhealthy. Detailed characteristics by overweight, obesity, and metabolic health were shown in Supplemental Table 1 (see Supplementary Material available online at http://dx.doi.org/10.1155/ 2016/4094876).

Metabolically unhealthy phenotypes showed significantly elevated mean values of total cholesterol, triglycerides, highdensity lipid cholesterol (HDLC), fasting glucose and insulin, HOMA IR, HbAlc, CRP, and blood pressure compared with metabolically healthy phenotypes. They were more likely to be older, cigarette smokers and unemployed, less well educated, and less likely to meet the recommended guidelines for physical activity of more than 600 MET-minutes/week. They had lower household income but more frequent family history of diabetes (all $P$ s $<0.05$ ). There was no difference in gender between metabolically unhealthy and healthy phenotypes (Table 1).

Overweight/obese phenotypes were also more likely to be older, less well educated, and cigarette smoking, had lower incomes but more frequent family history of diabetes, and showed significantly elevated mean values of total cholesterol, triglycerides, HDLC, fasting glucose and insulin, HOMA IR, HbAlc, CRP, blood pressure, BMI, WC, WHR, and body fat percentage compared with normal weight phenotypes (all $P s<0.05)$. There was no difference in gender, employment status, and physical activity between overweight/obese and normal weight phenotypes (Table 1).

Seventy-two participants of the cohort (2.2\%) were classified as metabolically unhealthy, normal weight. Compared with other three phenotypes, MUHNW subjects were more likely to be older, unemployed, and cigarette smoking, were least likely to meet the recommended guidelines for physical activity of more than 600 MET-minutes/week, and had least income and worst mean values in total cholesterol, SBP, and HbAlc (all $P$ s $<0.05)$ (Table 1).

A total of 878 (27.04\%) participants had diabetes (Table 2). Among the four phenotypes the MHNW phenotype had the lowest rates of diabetes $(12 \%)$ and the MUHOW phenotype had the highest $(40.3 \%)(P<0.0001)$ (Figure 1). Metabolically unhealthy subjects showed significantly higher diabetes prevalence than metabolically healthy subjects ( $40.3 \%$ versus $19.6 \% ; P<0.0001)$. Overweight/obese phenotypes showed significantly higher diabetes prevalence than normal weight phenotypes $(29.4 \%$ versus $15.2 \%$; $P<$ $0.0001)$.

Overweight/obese individuals showed an OR of having diabetes of 2.06 (95\% CI: 1.33-3.21) after adjusting for age and metabolic health. Poor metabolic health was positively related to the increased risk of diabetes ( $\mathrm{OR}=2.46 ; 95 \% \mathrm{CI}: 1.88-3.21$ ) after adjusting for age and overweight/obesity, suggesting that being metabolically unhealthy carried a higher risk for diabetes than being overweight/obese (2.46 versus 2.06). The risk of diabetes by categories of BMI and metabolic status is shown in Table 2. In a multivariable adjusted logistic regression model with diabetes as the dependent variable, MHOW subjects showed an OR of having diabetes of 2.25 (95\% CI 1.34-3.79), MUNW individuals showed an OR of 


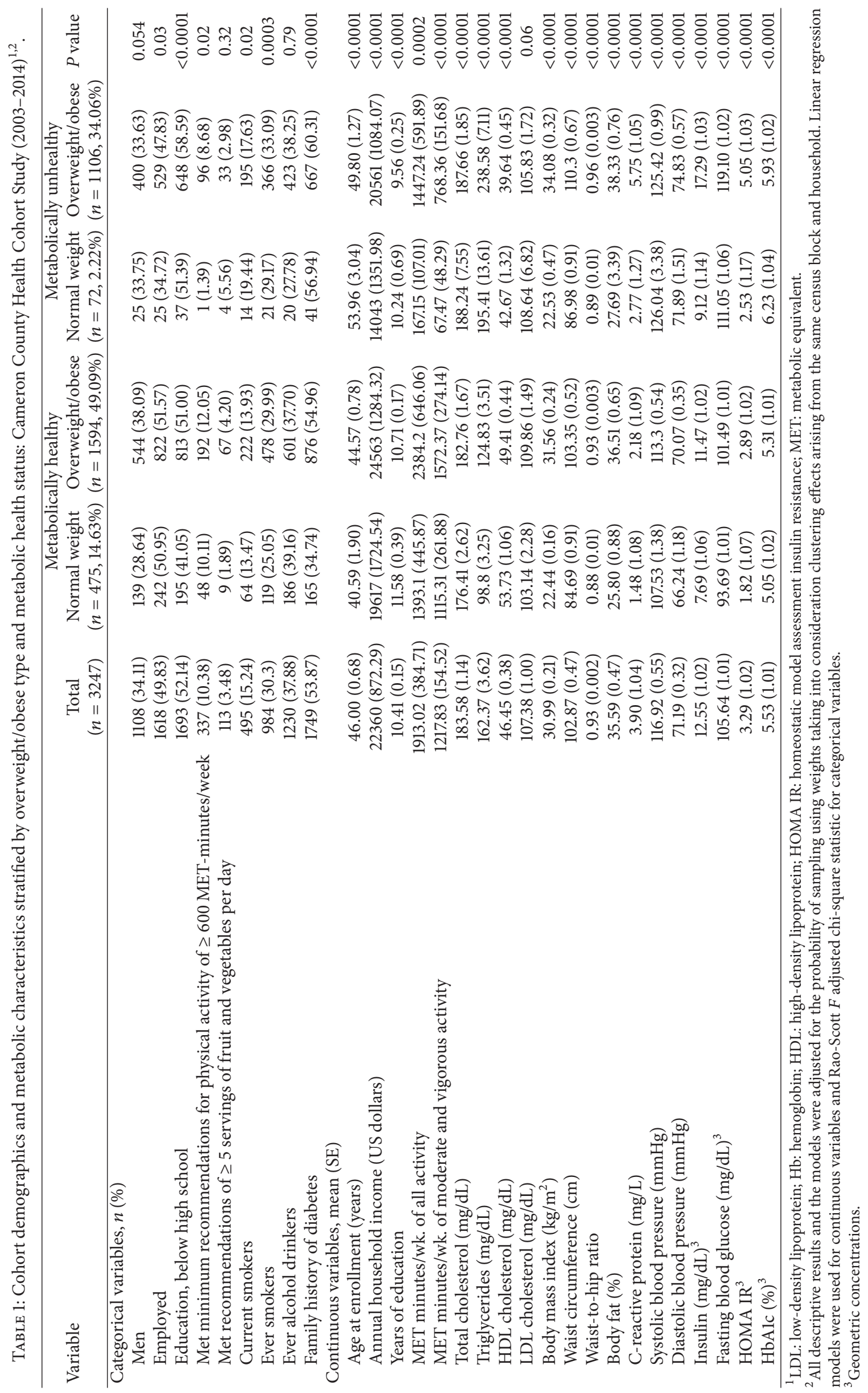


TABLE 2: Diabetes by overweight/obese type and metabolic health status.

\begin{tabular}{|c|c|c|c|c|c|}
\hline \multirow[b]{2}{*}{ Diabetes } & \multicolumn{2}{|c|}{ Metabolically healthy } & \multicolumn{2}{|c|}{ Metabolically unhealthy } & \multirow[b]{2}{*}{$P$ value } \\
\hline & $\begin{array}{l}\text { Normal weight } \\
\qquad(n=475)\end{array}$ & $\begin{array}{c}\text { Overweight/obese } \\
\quad(n=1594)\end{array}$ & $\begin{array}{l}\text { Normal weight } \\
\quad(n=72)\end{array}$ & $\begin{array}{l}\text { Overweight/obese } \\
\quad(n=1106)\end{array}$ & \\
\hline \multicolumn{6}{|l|}{ Primary analysis } \\
\hline \multicolumn{6}{|l|}{ Frequency } \\
\hline Yes $[n,(\%)]$ & $57(12.00)$ & $349(21.89)$ & $26(36.11)$ & $446(40.33)$ & $<0.0001^{1}$ \\
\hline No $[n,(\%)]$ & $401(84.42)$ & $1208(75.78)$ & $42(58.33)$ & $641(57.96)$ & \\
\hline \multicolumn{6}{|l|}{ Weighted OR (95\% CI) } \\
\hline Unadjusted model & Reference & $2.30(1.47,3.60)$ & $5.20(2.41,11.19)$ & $6.23(3.94,9.85)$ & $<0.0001^{2}$ \\
\hline Multivariable adjusted model $1^{3}$ & Reference & $2.25(1.34,3.79)$ & $3.78(1.57,9.09)$ & $5.39(3.16,9.20)$ & $<0.0001^{2}$ \\
\hline Multivariable adjusted model $2^{4}$ & Reference & $2.14(1.07,4.28)$ & $3.18(1.02,9.92)$ & $5.01(2.43,10.34)$ & $<0.0001^{2}$ \\
\hline \multicolumn{6}{|l|}{ Sensitivity analysis ${ }^{5}$} \\
\hline \multicolumn{6}{|l|}{ Frequency } \\
\hline Yes $[n,(\%)]$ & $41(9.53)$ & $145(12.49)$ & $42(35.90)$ & $650(42.24)$ & $<0.0001^{1}$ \\
\hline No $[n,(\%)]$ & $375(87.21)$ & $982(84.58)$ & $68(58.12)$ & $867(56.34)$ & \\
\hline \multicolumn{6}{|l|}{ Weighted OR (95\% CI) } \\
\hline Unadjusted model & Reference & $1.48(0.87,2.54)$ & $4.25(1.74,10.37)$ & $7.81(4.56,13.37)$ & $<0.0001^{2}$ \\
\hline Multivariable adjusted model $1^{3}$ & Reference & $1.47(0.83,2.61)$ & $2.93(1.04,8.23)$ & $6.25(3.54,11.02)$ & $<0.0001^{2}$ \\
\hline Multivariable adjusted model $2^{4}$ & Reference & $1.36(0.59,3.13)$ & $3.23(1.08,10.01)$ & $6.57(2.81,15.36)$ & $<0.0001^{2}$ \\
\hline
\end{tabular}

${ }^{1} F$ approximation of Rao-Scott design adjusted chi-square test $P$ value.

${ }^{2} P$ values from Wald chi-square test for the effect of overweight/obese phenotype.

${ }^{3}$ Adjusted for age at enrollment. Other covariates were not significant and not included in the final model. The models were adjusted for the probability of sampling using weights taking into consideration clustering effects arising from the same census block and household.

${ }^{4}$ Adjusted for age at enrollment and family history of diabetes. Restricted to the participants who had data for family history of diabetes $(n=2,234,68 \%)$.

${ }^{5}$ The definition of metabolically health included glucose component.

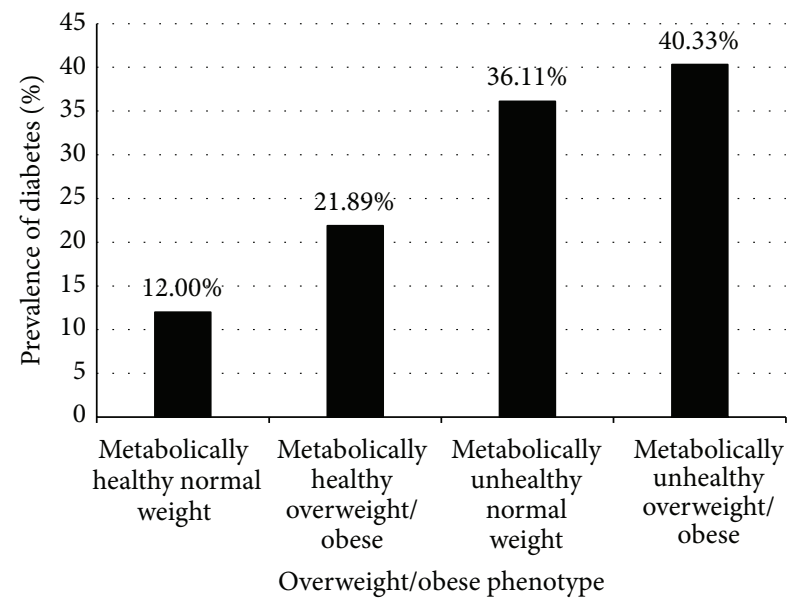

FIgURe 1: Prevalence of diabetes among Mexican Americans within each overweight/obese phenotype. Black bars indicate prevalence rate. Diabetes prevalence was different by overweight/obese phenotype $(P<0.0001)$.

3.78 (95\% CI 1.57-9.09), and MUHOW subjects showed an OR of 5.39 (95\% CI 3.16-9.20) after adjusting for confounding factors with the MHNW phenotype as the reference (Table 2). The biggest effect comes from being metabolically unhealthy and normal weight: the adjusted odds ratio for this group compared to the metabolically healthy and normal weight one is 3.8 , while the OR for metabolically unhealthy and normal weight compared to metabolically healthy and normal weight is just over 2 (Table 2). The addition of metabolically unhealthy phenotypes to obesity increases the OR for diabetes to over 5-fold (Table 2). The ORs for the risk of diabetes were greater than 1 for the metabolically unhealthy phenotype and were much higher than the ORs for MHNW and MHOW phenotypes: the difference of ORs between MUHNW and MHNW phenotypes was 278\%; and the difference of ORs between MUHOW and MHOW phenotypes was $140 \%$, while the difference of ORs between MHOW and MHNW phenotypes was $125 \%$, and the difference of ORs between MUHNW and MUHOW phenotypes was $43 \%$. These comparisons suggested that the risk of diabetes in metabolically unhealthy phenotype was higher than healthy phenotype in any category of BMI, and the metabolic health is more important than simple overweight/obesity. Restricted to the participants who had available data for family history of diabetes $(68 \%)$, the ORs in each phenotype were not materially changed. When fasting blood glucose $>100 \mathrm{mg} / \mathrm{dL}$ or being on hypoglycemic medication was included as a component for the definition of metabolic health in the sensitivity analysis, the correlation was not significant for the MHOW phenotype, the correlation for the MUHNW phenotype remained similar, and the correlation for the MUHOW phenotype became slightly stronger. The sensitivity analysis results further suggested that the metabolic health is more important than simple overweight/obesity. Supplemental Table 2 further showed the risk of diabetes by overweight, obese, and metabolic status. Similar patterns as 

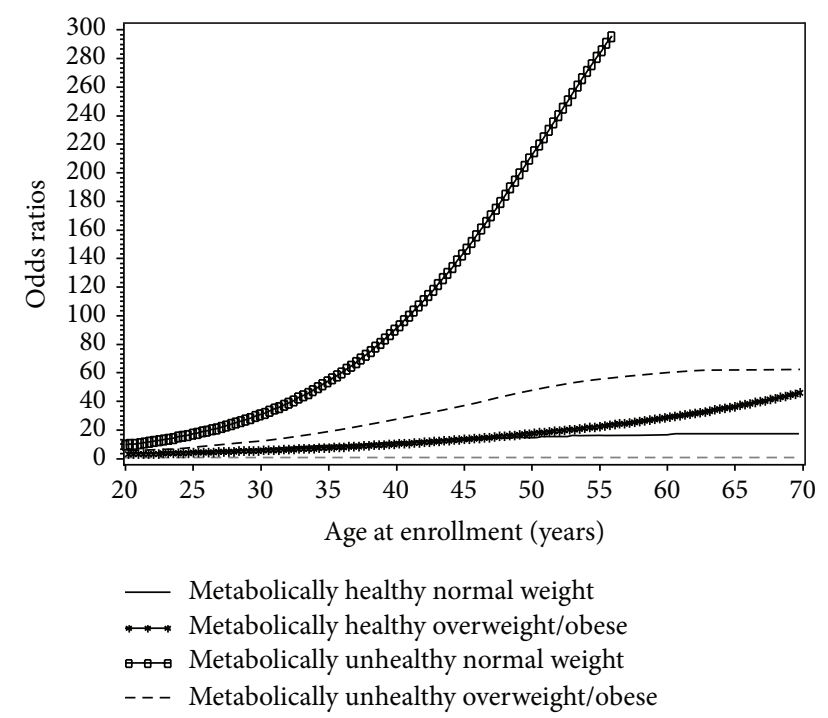

FIgURE 2: Smoothed plot for odds ratios (ORs) of the diabetes risk according to age at enrollment. Subjects were divided into four phenotypes according to overweight/obese phenotype and metabolic health status. The ORs were estimated by using the restricted cubic spline logistic regression models with knots placed at the 5th, 50th, and 95th percentiles of age at enrollment. The models were adjusted for the probability of sampling using weights taking into consideration clustering effects arising from the same census block and household. The linear correlation between age at enrollment and the risk of diabetes in each phenotype was significant $(P=0.02,0.0001,0.04$, and $<0.0001$ for phenotypes with metabolically healthy normal weight, metabolically healthy overweight/obesity, metabolically unhealthy normal weight, and metabolically unhealthy overweight/obesity, resp.).

in Table 2 indicated that the risk of diabetes in metabolically unhealthy phenotype was higher than healthy phenotype, and the metabolic health is more important than simple overweight or obesity.

Figure 2 visually depicts the shape of the correlation between age and diabetes risk in four phenotypes after adjusting for potential confounding variables in a restricted cubic spline model. Age was positively and approximately linearly associated with the risk for diabetes in each phenotype (all $P$ s $<0.05)$. Metabolically unhealthy phenotypes had higher ORs than their corresponding counterparts in any category of BMI, and MUHNW phenotype had the highest ORs in the four phenotypes. MHOW subjects had higher ORs than MHNW subjects, and the latter had the lowest ORs in the four phenotypes.

\section{Discussion}

In a Mexican American cohort metabolically unhealthy subjects showed significantly increased risk for diabetes compared with metabolically healthy subjects in any category of BMI. Compared with the metabolically healthy normal weight participants (MHNW), the metabolically unhealthy, regardless of their BMI (MUHNW and MUHOW), and the metabolically healthy obese (MHOW) phenotypes had significantly increased risk of diabetes. MUHNW individuals had a fourfold increased risk and MUHOW individuals had a fivefold increased risk for diabetes compared with the MHNW phenotype. Cubic spline interpolation showed that the risk of diabetes with age was higher in metabolically unhealthy phenotype than metabolically healthy phenotype in any category of BMI. The significance of these observations is that poor metabolic health puts the individual at greater risk of diabetes than obesity alone.

A high proportion of the Mexican Americans in our population are metabolically unhealthy (36.3\%) but over half $(59.9 \%)$ if the definition of metabolic health includes a glucose component. Because we wanted to examine the relationship of diabetes, a disease of glucose metabolism, we excluded that from our criteria for metabolic abnormalities, yet metabolic abnormalities remain the major association with diabetes. Others have shown a high prevalence of metabolically unhealthy Mexican Americans (30\%) [6]. In general, Mexican Americans also have high prevalence of diabetes, overweight/obesity, and metabolic abnormalities compared to non-Hispanic Whites [14-16]. Using both logistic regression and cubic spline models, being metabolically unhealthy posed a significantly higher risk for diabetes than being overweight. Similar observations have been made in a prospective cohort study in 6,748 Koreans [5], although the results were not all statistically significant among different overweight/obese and metabolic health phenotypes [5]. Furthermore, we found that metabolically unhealthy overweight/obese individuals were more likely to develop incident diabetes compared with their normal weight counterparts which was consistent with other findings [4-6]. An important element here is the identification of those with metabolic risk factors but who are not obese since they are at high risk for diabetes but less likely to be identified early and provided prevention education. These individuals may well be overlooked in screening programs.

It is not surprising that poor metabolic health puts the individual at a higher risk of diabetes than overweight/obesity alone. In our study, metabolically unhealthy phenotype tended to do less exercise and had lower education and less income level but had increased cigarette smoking compared with metabolically healthy phenotypes. In particular, the MUHNW phenotype showed the lowest proportion of subjects who met minimum recommendations for physical activity of $\geq 600$ MET-minutes/week despite their normal weight, although physical activity was not significant in multivariable analysis for the risk of diabetes. Because physical activity was not correlated with diabetes risk $(P=0.18)$ and metabolic health $(P=0.07)$ in logistic regression models and it was not statistically significant in the multivariable adjusted model $(P=0.46)$, it was not adjusted for in the final model.

Metabolically unhealthy phenotype had higher markers of inflammation which may be the key underlying pathology. Metabolically unhealthy subjects showed significantly higher levels of CRP compared with their metabolically healthy counterparts, consistent with the known role of systemic inflammation in the risk of diabetes. Several conditions that are driven by inflammatory processes are also associated with diabetes, including rheumatoid arthritis, gout, psoriasis, 
and Crohn's disease, and various anti-inflammatory drugs have been approved or are in late stages of development for the treatment of these conditions [26]. Another important difference between metabolically healthy and unhealthy phenotypes was markedly higher dyslipidemia, measured as hypertriglyceridemia and low HDL-C, fasting glucose, high blood pressure, or insulin resistance observed in the metabolically unhealthy phenotype. These results suggest the importance of lifestyle modification and control of systemic inflammation in maintaining metabolic health and normoglycemia, not the simple reduction in body weight, although physical activity was not significant in multivariable analysis for the risk of diabetes.

Our study found a positive dose-response with approximately linear relationship between age and the risk of diabetes in each phenotype, stratified by overweight/obesity and metabolic health. Metabolically unhealthy phenotypes had higher risk than their corresponding counterparts in any category of BMI, and MUHNW phenotype had the highest ORs in the four phenotypes. To our knowledge, this is the first study to find the risk of diabetes with age higher in metabolically unhealthy phenotypes than in metabolically healthy phenotypes in any category of BMI. The Korean Healthy Twin Study $(n=2,687)$ reported that the risk of diabetes was 4.4-fold higher in MUHNW individuals than in MHNW individuals and 3.3-fold higher in MUHOW subjects than in MHNW subjects [27]. Despite a normal weight identical to the MHNW subjects, MUHNW subjects in our study presented an increased fasting serum insulin and blood glucose, HOMA IR, and HbAlc. This phenomenon may be associated with impaired insulin sensitivity (euglycemic hyperinsulinemic clamp or oral glucose tolerance test) $[11,28]$. Although the mechanism is still not clear, at least we are now aware that the MUHNW individuals are a target population in which to identify and to prevent diabetes [27].

Previous studies have suggested that metabolic healthy overweight/obesity was not a benign condition [4-6]. MHOW individuals may be more likely to have diabetes compared with metabolically healthy normal weight peers $[4,6]$. Our study found that MHOW subjects had a significant 2.14-fold elevated risk of diabetes (Table 2) and they were more likely to do this before 50 years of age compared with their MHNW counterparts (Figure 1). However, in our sensitivity analysis including fasting glucose as a component for the definition of metabolic health, the risk of diabetes was not significant for the MHOW phenotype. Clearly, the risk of diabetes in MHOW populations needs to be further investigated.

Although a small proportion of the subjects (2.22\%) were classified as metabolically unhealthy normal weight phenotype, their risk for diabetes was higher than MHNW and MHOW (Table 2) and they had the highest ORs for the correlation between age and diabetes risk in the four phenotypes (Figure 2). The proportion might be increased if there is no effort to protect this population, because these subjects are not overweight or obese and they may escape detection and therefore not benefit from adequate treatment or prevention measures. Furthermore, the interventions to boost metabolic health involved changes in lifestyles such as diet, exercise, and behavior (smoking and others) which may not be related to overweight/obesity [29]. Therefore, it is very important to make efforts on improving metabolic health in any categories of BMI. The MUHNW phenotype needs to be included within the scope of prevention and control, but should not be ignored.

There are some methodological limitations in our research. The study was cross-sectional in design; thus, only association but not causal relationship may be inferred. Prospective studies are needed to further investigate whether metabolic health is more important than overweight/obesity alone. Our longitudinal data currently being collected will provide that opportunity. We could not completely rule out the possibility of residual confounding due to unmeasured or inadequately measured covariates such as the missing values with some variables.

This study had several strengths. First, this is a general population-based randomly selected Mexican American cohort, thus avoiding bias inherent in studies drawn from clinic populations or other nonrandomly selected populations with established disease or mixed ethnicity. Second, detailed information on a wide range of factors related to diabetes was available, allowing us to get a relatively comprehensive analysis of the affecting factors. Third, cubic spline interpolation was used to compare the dose-response correlation between age and the diabetes risk in different metabolic health and overweight/obese phenotypes and suggested the importance of metabolic health compared to overweight/obesity for the risk of diabetes associated with age. Finally, published studies generally only compared the disease status between obesity (BMI $\geq 30 \mathrm{~kg} / \mathrm{m}^{2}$ ) and normal weight phenotypes stratified by the metabolic health status, while the risk of diseases for the phenotype with BMI 25$30 \mathrm{~kg} / \mathrm{m}^{2}$ was neglected $[6,30]$. However, previous studies $[2,3]$ and our study found that overweight/obese individuals $\left(\mathrm{BMI} \geq 25 \mathrm{~kg} / \mathrm{m}^{2}\right)$ were at a higher risk of diabetes compared with normal weight individuals.

In conclusion, in our cohort those who had more than two markers indicating unhealthy metabolism had statistically higher prevalence and odds of having diabetes compared to those with healthy metabolism suggesting a higher risk of diabetes adjusting for age, gender or BMI, and overweight/obese. Therefore, being metabolically unhealthy is likely more important for the risk of diabetes than simply being overweight/obese. Efforts need to be focused on improving metabolic health in all categories of BMI. Early lifestyle intervention in these populations is likely to be more effective than simple weight loss.

\section{Conflict of Interests}

The authors declare that there is no conflict of interests regarding the publication of this paper.

\section{Authors' Contribution}

The authors' responsibilities were as follows: Joseph B. McCormick and Susan P. Fisher-Hoch designed research; 
Joseph B. McCormick, Susan P. Fisher-Hoch, and Belinda Reninger conducted research; Shenghui Wu analyzed data; Kristina Vatcheva provided statistical support; Shenghui Wu, Joseph B. McCormick, and Susan P. Fisher-Hoch wrote the paper. All authors read and approved the final paper.

\section{Acknowledgments}

The authors thank their cohort team, particularly, Rocio Uribe (Brownsville), Becky Erazo (Laredo), Ariana Garza (Harlingen), and their teams, who recruited and documented the participants. The authors also thank Marcela Morris and other laboratory staff for their contributions and Christina Villarreal for administrative support. The authors thank Valley Baptist Medical Center, Brownsville, Texas, for providing them space for their Center for Clinical and Translational Science Clinical Research Unit. The authors also thank the community of Brownsville, Laredo, and Harlingen and the participants who so willingly participated in this study in their city. This work was supported by MD000170 P20 funded by the National Center on Minority Health and Health Disparities, the Centers for Translational Science Award 1U54RR023417-01 from the National Center, and the Centers for Disease Control Award RO1 DP000210-01 for Research Resources.

\section{References}

[1] M. Ng, T. Fleming, M. Robinson et al., "Global, regional, and national prevalence of overweight and obesity in children and adults during 1980-2013: a systematic analysis for the Global Burden of Disease Study 2013," The Lancet, vol. 384, no. 9945, pp. 766-781, 2014.

[2] G. Whitlock, S. Lewington, P. Sherliker et al., "Body-mass index and cause-specific mortality in 900000 adults: collaborative analyses of 57 prospective studies," The Lancet, vol. 373, no. 9669, pp. 1083-1096, 2009.

[3] J. A. Bell, M. Kivimaki, and M. Hamer, "Metabolically healthy obesity and risk of incident type 2 diabetes: a meta-analysis of prospective cohort studies," Obesity Reviews, vol. 15, no. 6, pp. 504-515, 2014.

[4] Y. Heianza, K. Kato, S. Kodama et al., "Risk of the development of Type 2 diabetes in relation to overall obesity, abdominal obesity and the clustering of metabolic abnormalities in Japanese individuals: does metabolically healthy overweight really exist? The Niigata Wellness study," Diabetic Medicine, vol. 32, no. 5, pp. 665-672, 2015.

[5] E. Rhee, M. K. Lee, J. D. Kim et al., "Metabolic health is a more important determinant for diabetes development than simple obesity: a 4-year retrospective longitudinal study," PLOS ONE, vol. 9, no. 5, Article ID e98369, 2014.

[6] K. Aung, C. Lorenzo, M. A. Hinojosa, and S. M. Haffner, "Risk of developing diabetes and cardiovascular disease in metabolically unhealthy normal-weight and metabolically healthy obese individuals," Journal of Clinical Endocrinology and Metabolism, vol. 99, no. 2, pp. 462-468, 2014.

[7] N. Ruderman, D. Chisholm, X. Pi-Sunyer, and S. Schneider, "The metabolically obese, normal-weight individual revisited," Diabetes, vol. 47, no. 5, pp. 699-713, 1998.
[8] N. B. Ruderman, S. H. Schneider, and P. Berchtold, "The 'metabolically-obese,' normal-weight individual," American Journal of Clinical Nutrition, vol. 34, no. 8, pp. 1617-1621, 1981.

[9] Y.-W. Park, S. Zhu, L. Palaniappan, S. Heshka, M. R. Carnethon, and S. B. Heymsfield, "The metabolic syndrome: prevalence and associated risk factor findings in the US population from the Third National Health and Nutrition Examination Survey, 1988-1994," Archives of Internal Medicine, vol. 163, no. 4, pp. 427-436, 2003.

[10] S. T. Laing, B. Smulevitz, K. P. Vatcheva et al., "Subclinical atherosclerosis and obesity phenotypes among Mexican Americans," Journal of the American Heart Association, vol. 4, no. 3, Article ID e001540, 2015.

[11] F. Conus, R. Rabasa-Lhoret, and F. Péronnet, "Characteristics of metabolically obese normal-weight (MONW) subjects," Applied Physiology, Nutrition, and Metabolism, vol. 32, no. 1, pp. 4-12, 2007.

[12] K. M. Choi, H. J. Cho, H. Y. Choi et al., "Higher mortality in metabolically obese normal-weight people than in metabolically healthy obese subjects in elderly Koreans," Clinical Endocrinology, vol. 79, no. 3, pp. 364-370, 2013.

[13] E. S. Ford, C. Li, and N. Sattar, "Metabolic syndrome and incident diabetes: current state of the evidence," Diabetes Care, vol. 31, no. 9, pp. 1898-1904, 2008.

[14] M. I. Harris, K. M. Flegal, C. C. Cowie et al., "Prevalence of diabetes, impaired fasting glucose, and impaired glucose tolerance in U.S. Adults: the Third National Health and Nutrition Examination Survey, 1988-1994," Diabetes Care, vol. 21, no. 4, pp. 518-524, 1998.

[15] K. M. Flegal, C. L. Ogden, and M. D. Carroll, "Prevalence and trends in overweight in Mexican-American adults and children," Nutrition Reviews, vol. 62, supplement 2, no. 7, pp. S144-S148, 2004.

[16] L. Razzouk and P. Muntner, "Ethnic, gender, and age-related differences in patients with the metabolic syndrome," Current Hypertension Reports, vol. 11, no. 2, pp. 127-132, 2009.

[17] S. P. Fisher-Hoch, A. R. Rentfro, J. J. Salinas et al., "Socioeconomic status and prevalence of obesity and diabetes in a Mexican American community, Cameron County, Texas, 2004-2007," Preventing chronic disease, vol. 7, no. 3, article A53, 2010.

[18] S. P. Fisher-Hoch, K. P. Vatcheva, S. T. Laing et al., "Missed opportunities for diagnosis and treatment of diabetes, hypertension, and hypercholesterolemia in a Mexican American population, Cameron County Hispanic Cohort, 2003-2008," Preventing Chronic Disease, vol. 9, Article ID 110298, 2012.

[19] S. S. Sun, W. C. Chumlea, S. B. Heymsfield et al., "Development of bioelectrical impedance analysis prediction equations for body composition with the use of a multicomponent model for use in epidemiologic surveys," The American Journal of Clinical Nutrition, vol. 77, no. 2, pp. 331-340, 2003.

[20] C. L. Craig, A. L. Marshall, M. Sjostrom et al., "International physical activity questionnaire: 12 -country reliability and validity," Medicine \& Science in Sports \& Exercise, vol. 35, no. 8, pp. 1381-1395, 2003.

[21] D. R. Matthews, J. P. Hosker, A. S. Rudenski, B. A. Naylor, D. F. Treacher, and R. C. Turner, "Homeostasis model assessment: insulin resistance and $\beta$-cell function from fasting plasma glucose and insulin concentrations in man," Diabetologia, vol. 28, no. 7, pp. 412-419, 1985.

[22] S. M. Grundy, J. I. Cleeman, S. R. Daniels et al., "Diagnosis and management of the metabolic syndrome: an American Heart 
Association/National Heart, Lung, and Blood Institute scientific statement," Circulation, vol. 112, no. 17, pp. 2735-2752, 2005.

[23] R. P. Wildman, P. Muntner, K. Reynolds et al., "The obese without cardiometabolic risk factor clustering and the normal weight with cardiometabolic risk factor clustering: prevalence and correlates of 2 phenotypes among the US population (NHANES 1999-2004)," Archives of Internal Medicine, vol. 168, no. 15, pp. 1617-1624, 2008.

[24] American Diabetes Association, "Standards of medical care in diabetes-2010," Diabetes Care, vol. 33, supplement 1, pp. S11S61, 2010.

[25] F. J. Harrell Jr., Regression Modeling Strategies: With Applications to Linear Models, Logistic Regression, and Survival Analysis, Springer, New York, NY, USA, 2001.

[26] M. Y. Donath, "Targeting inflammation in the treatment of type 2 diabetes: time to start," Nature Reviews Drug Discovery, vol. 13, no. 6, pp. 465-476, 2014.

[27] Y.-M. Song, J. Sung, and K. Lee, "Genetic and environmental relationships of metabolic and weight phenotypes to metabolic syndrome and diabetes: the Healthy Twin Study," Metabolic Syndrome and Related Disorders, vol. 13, no. 1, pp. 36-44, 2015.

[28] R. V. Dvorak, W. F. DeNino, P. A. Ades, and E. T. Poehlman, "Phenotypic characteristics associated with insulin resistance in metabolically obese but normal-weight young women," Diabetes, vol. 48, no. 11, pp. 2210-2214, 1999.

[29] N. Bassi, I. Karagodin, S. Wang et al., "Lifestyle modification for metabolic syndrome: a systematic review," The American Journal of Medicine, vol. 127, no. 12, pp. 1242.e1-1242.e10, 2014.

[30] S. L. Appleton, C. J. Seaborn, R. Visvanathan et al., "Diabetes and cardiovascular disease outcomes in the metabolically healthy obese phenotype: a cohort study," Diabetes Care, vol. 36, no. 8, pp. 2388-2394, 2013. 


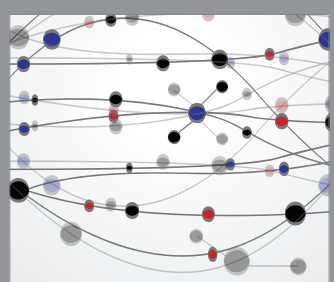

The Scientific World Journal
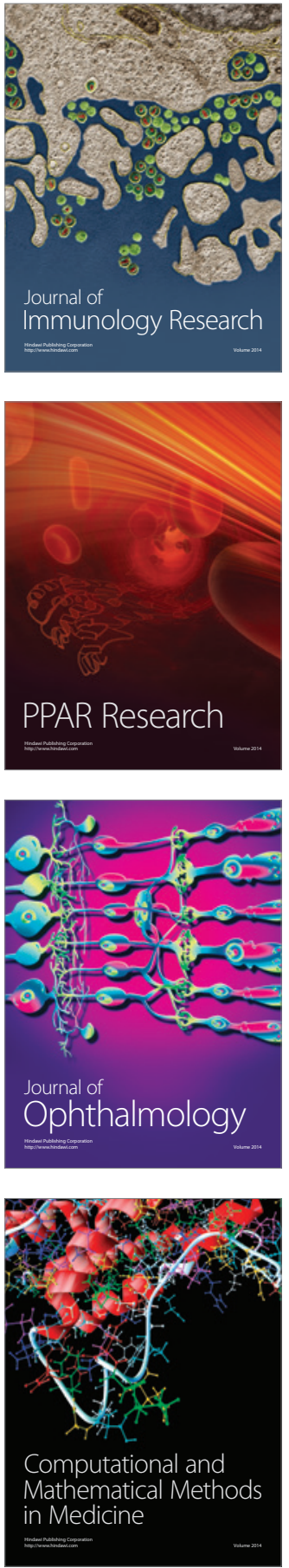

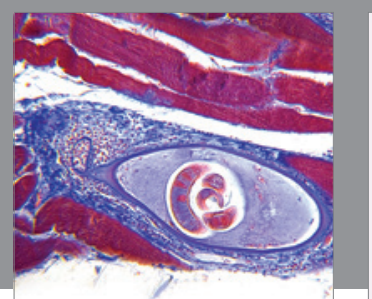

Gastroenterology Research and Practice

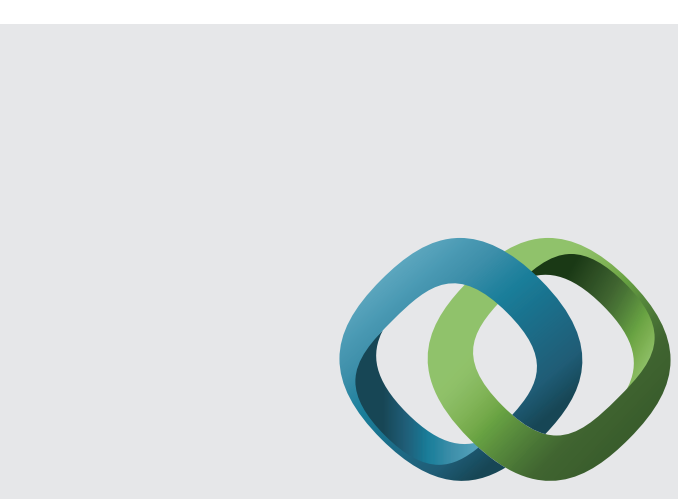

\section{Hindawi}

Submit your manuscripts at

http://www.hindawi.com
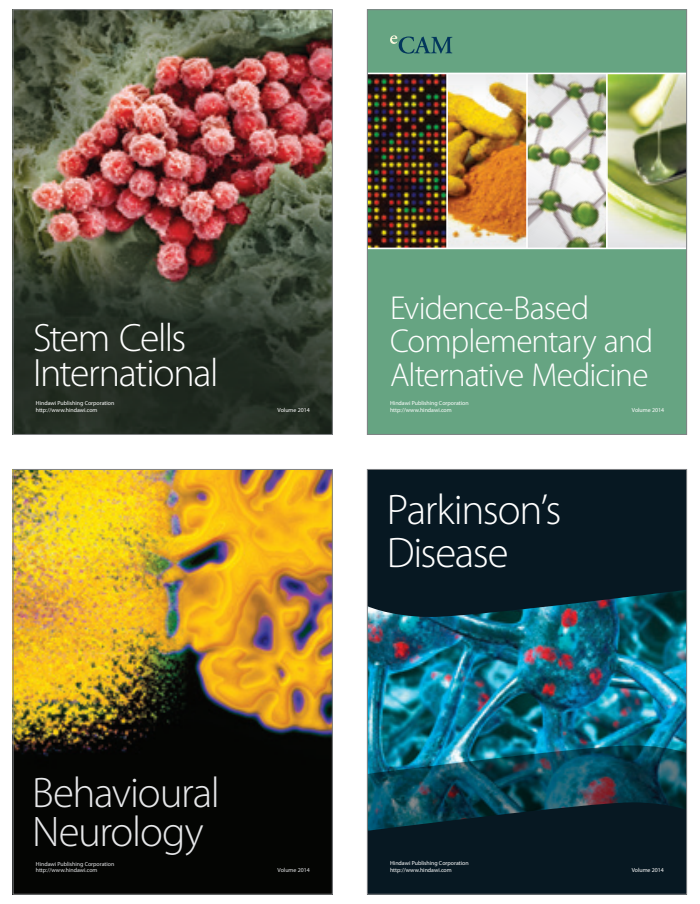
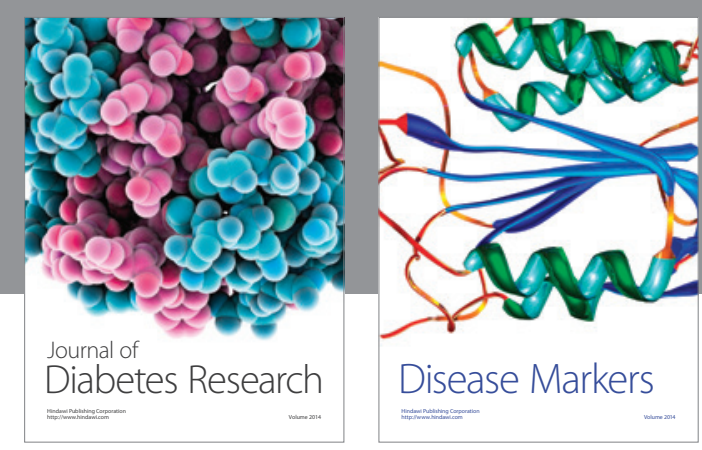

Disease Markers
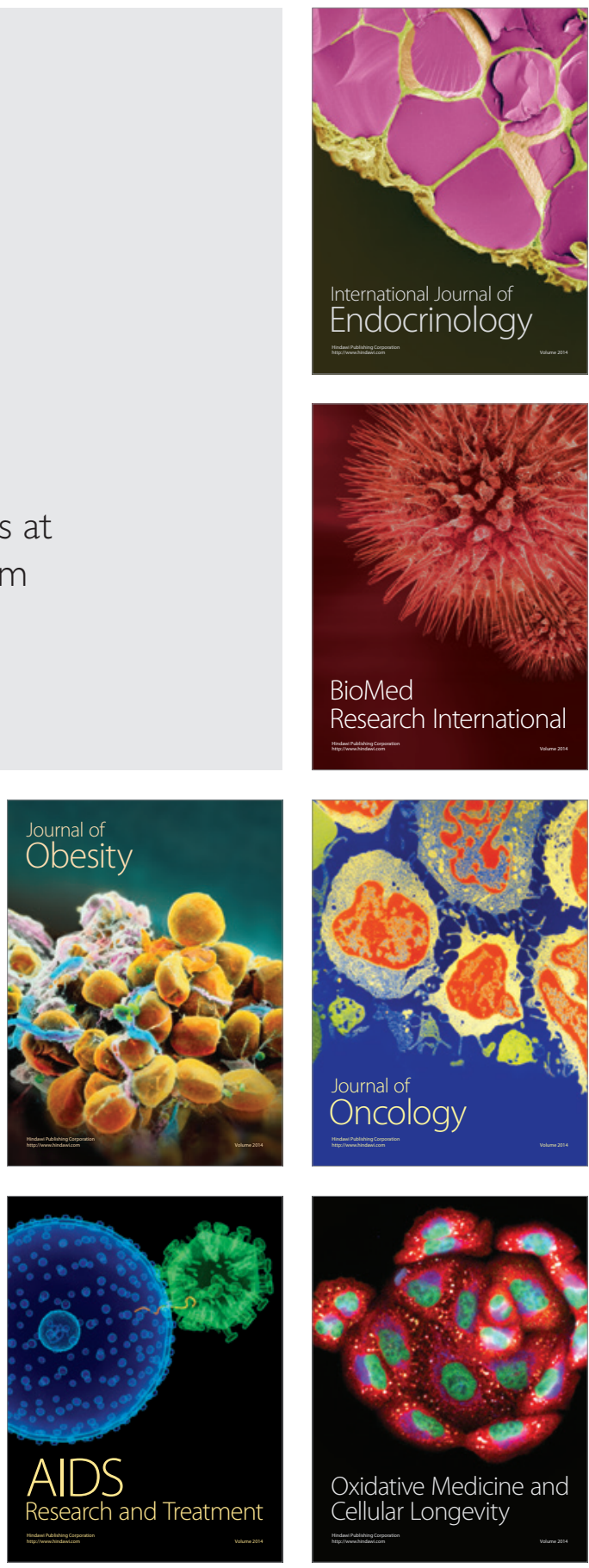